\title{
Direct Observation of Dynamical Quantum Phase Transitions in an Interacting Many-Body System
}

\author{
P. Jurcevic, ${ }^{1,2}$ H. Shen, ${ }^{1}$ P. Hauke, ${ }^{1,3}$ C. Maier,${ }^{1,2}$ T. Brydges, ${ }^{1,2}$ C. Hempel,,${ }^{1, *}$ B. P. Lanyon, ${ }^{1,2}$ \\ M. Heyl, ${ }^{4,5}$ R. Blatt, ${ }^{1,2}$ and C. F. Roos ${ }^{1,2}$ \\ ${ }^{1}$ Institut für Quantenoptik und Quanteninformation, Österreichische Akademie der Wissenschaften, \\ Technikerstr. 21A, 6020 Innsbruck, Austria \\ ${ }^{2}$ Institut für Experimentalphysik, Universität Innsbruck, Technikerstrasse 25, 6020 Innsbruck, Austria \\ ${ }^{3}$ Institut für Theoretische Physik, Universität Innsbruck, Technikerstrasse 25, 6020 Innsbruck, Austria \\ ${ }^{4}$ Max-Planck-Institut für Physik komplexer Systeme, 01187 Dresden, Germany \\ ${ }^{5}$ Physik Department, Technische Universität München, 85747 Garching, Germany
}

(Received 10 March 2017; published 21 August 2017)

\begin{abstract}
The theory of phase transitions represents a central concept for the characterization of equilibrium matter. In this work we study experimentally an extension of this theory to the nonequilibrium dynamical regime termed dynamical quantum phase transitions (DQPTs). We investigate and measure DQPTs in a string of ions simulating interacting transverse-field Ising models. During the nonequilibrium dynamics induced by a quantum quench we show for strings of up to 10 ions the direct detection of DQPTs by revealing nonanalytic behavior in time. Moreover, we provide a link between DQPTs and the dynamics of other quantities such as the magnetization, and we establish a connection between DQPTs and entanglement production.
\end{abstract}

DOI: 10.1103/PhysRevLett.119.080501

Today, the equilibrium properties of quantum matter are theoretically described with great success. Yet, in recent years pioneering experiments have created quantum states beyond this equilibrium paradigm [1,2]. Thanks to this progress, it is now possible to experimentally study exotic phenomena such as many-body localization $[3,4]$, prethermalization $[5,6]$, particle-antiparticle production in the lattice Schwinger model [7], and light-induced superconductivity [8]. Understanding general properties of such nonequilibrium quantum states provides a significant challenge, calling for concepts that extend important principles such as universality to the nonequilibrium realm. A general approach towards this major goal is the theory of dynamical quantum phase transitions (DQPTs) [9], which extends the concept of phase transitions and thus universality to the nonequilibrium regime. In this Letter, we directly observe the defining real-time nonanalyticities at DQPTs in a trapped-ion quantum simulator for interacting transverse-field Ising models. Moreover, we provide a link between DQPTs and the dynamics of other relevant quantities such as the magnetization, and we establish a connection between DQPTs and entanglement production.

Statistical mechanics and thermodynamics provide us with an excellent understanding of equilibrium quantum many-body systems. A key concept in this framework is the canonical partition function $Z(T)=\operatorname{Tr}\left(e^{-H / k_{B} T}\right)$, with $T$ being the temperature, $k_{B}$ the Boltzmann constant, and $H$ the system Hamiltonian. The partition function encodes thermodynamics via the free-energy density $f=-\left(k_{B} T / N\right) \log$ $[Z(T)]$, where $N$ denotes the number of degrees of freedom.
A phase transition, i.e., a sudden change of macroscopic behavior, is associated with a nonanalytical behavior of $f$ as a function of temperature or another control parameter $g$ such as an external magnetic field. QPTs [10] occur when $T$ is kept at absolute 0, where the system's ground-state properties undergo a nonanalytic change as a function of $g$ [see Fig. 1(a)]. Scenarios where boundary conditions are essential, such as for the Casimir effect, can be studied by boundary partition functions $Z_{B}=\left\langle\psi_{0}\left|e^{-R H}\right| \psi_{0}\right\rangle$, where $\left|\psi_{0}\right\rangle$ encodes the spatial boundary conditions on two ends of the system at distance $R$ [11].

Out of equilibrium, a partition function in the conventional sense cannot be formulated. Yet DQPTs, where physical quantities show nonanalytic behavior as a function of time, can still occur [9]. Within the theory of DQPTs, the formal role of the partition function is taken by the complex Loschmidt amplitude $\mathcal{G}(t)=\left\langle\psi_{0}\left|e^{-i H t}\right| \psi_{0}\right\rangle$, where $H$ is the Hamiltonian driving the time evolution and $\left|\psi_{0}\right\rangle$ denotes a pure quantum state, e.g., the ground state of some initial Hamiltonian $H_{0}$. Introducing a dynamical, complex counterpart to the free-energy density, $\gamma(t)=-N^{-1} \log [\mathcal{G}(t)]$, a DQPT is defined as a nonanalytic behavior in $\gamma(t)$ occurring in the thermodynamic limit as a function of time $t$ instead of a control parameter [see Fig. 1(b)]. Theoretically, it is not yet settled under which circumstances DQPTs occur, though a necessary condition seems to be a sufficiently strong perturbation. This can often be achieved when a system parameter is quenched across an underlying equilibrium critical point. However, it is important to emphasize that DQPTs constitute a distinct 

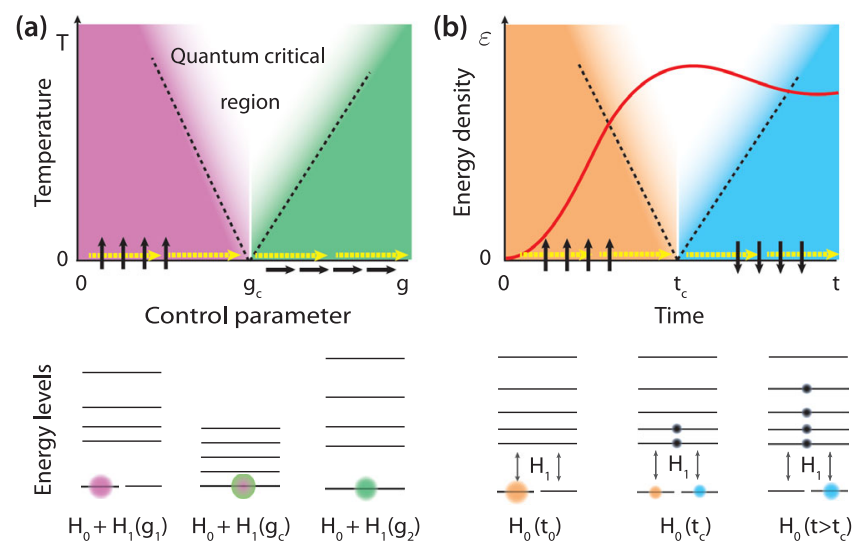

FIG. 1. Schematic comparison of conventional and dynamical quantum phase transitions. (a) Equilibrium temperature-control parameter phase diagram. A quantum phase transition (QPT) is a continuous phase transition occurring at $T=0$ and separating two phases, e.g., a ferromagnet for $g<g_{c}$ from a paramagnet for $g>g_{c}$ (black arrows). At $g_{c}$, physical quantities become nonanalytic upon varying $g$ (yellow arrows), triggered by a change in the spectrum of the system Hamiltonian $H=H_{0}+H_{1}(g)$, which becomes gapless as indicated by the schematic energy-level structure. Though only occurring at $T=0$, QPTs control the system's properties also in the quantum critical region at $T>0$. (b) Dynamics in the energy density-time plane. A DQPT occurs along the $\varepsilon=0$ axis at $t=t_{c}$, separating two regimes of, e.g., opposite magnetization (black arrows). The DQPT is not associated with a change in the spectrum but with an incisive redistribution of occupations between the eigenstates of the initial Hamiltonian $H_{0}$, induced by the perturbation $H_{1}$. In the present experiment, $H_{0}$ exhibits two degenerate ground states of opposite magnetization, and the DQPT is caused by a sudden change of the low-energy occupations from one of the two ground states to the other. Though the mean energy density (red line), where many observables acquire their dominant contribution, lies at $\varepsilon>0$, the nonequilibrium dynamics of observables can still be controlled by the underlying DQPT (white space).

critical phenomenon not in one-to-one correspondence with equilibrium phase transitions [compare Figs. 1(a) and 1(b)]. Still, many essential concepts such as universality and scaling [12] as well as robustness [13-15] carry over to them. Moreover, order parameters have recently been identified theoretically $[16,17]$. Very recently, a dynamical topological order parameter for DQPTs has been observed in a system of ultracold atoms in an optical lattice [18].

Here, we report on the first direct observation of a DQPT by resolving the nonanalyticity in the evolution of a quantum many-body system at a DQPT. We demonstrate that the DQPTs are robust against modifications of microscopic details of the underlying Hamiltonian. Moreover, we provide a physical picture of how an underlying DQPT controls the dynamics of other quantities such as the magnetization and, finally, we establish a connection between the occurrence of a DQPT and entanglement production.
We study DQPTs in a trapped-ion quantum simulator, realizing the dynamics of an effective transverse-field Ising Hamiltonian [19-21],

$$
\begin{gathered}
H_{\text {Ising }}=H_{0}+H_{1}, \\
H_{0}=-\hbar \sum_{i<j}^{N} J_{i j} \sigma_{i}^{x} \sigma_{j}^{x}, \quad H_{1}=-\hbar B \sum_{i}^{N} \sigma_{i}^{z},
\end{gathered}
$$

with $\sigma_{i}$ being Pauli spin operators on sites $i=1, \ldots, N$. The coupling matrix $J_{i j}>0$ has approximately a spatial powerlaw dependence, $J_{i j} \sim J_{i, i+1} /|i-j|^{\alpha}$, with $0<\alpha<3$ as a tunable parameter. The Hamiltonian $H_{0}$ exhibits spontaneous symmetry breaking with two degenerate ground states, $|\Rightarrow\rangle$ and $|\Leftarrow\rangle$, with $\sigma_{i}^{x}|\Rightarrow\rangle=|\Rightarrow\rangle$ and $\sigma_{i}^{x}|\Leftarrow\rangle=-|\Leftarrow\rangle \forall i$, respectively. Recently, DQPTs in such Ising models have been studied theoretically $[22,23]$. In the experiment, the (pseudo-) spins are realized in two electronic states, e.g., $\left|S_{1 / 2}, m\right\rangle \equiv|\downarrow\rangle_{z}$ and $\left|D_{5 / 2}, m^{\prime}\right\rangle \equiv|\uparrow\rangle_{z}$, of ${ }^{40} \mathrm{Ca}^{+}$ions arranged in a linear string, and the encoded spins are coupled and manipulated with lasers (see Supplemental Material [24]).

In our experiment, we adopt the following general protocol. First, the ion chain is initialized in one of the two ground states of the initial Hamiltonian $H_{0}$, say $\left|\psi_{0}\right\rangle=|\Rightarrow\rangle$. At time $t=0$, the Hamiltonian is suddenly switched to $H=H_{0}+H_{1}$, and the system state evolves to $|\psi(t)\rangle=e^{-i H t}\left|\psi_{0}\right\rangle$, realizing a so-called quantum quench [1]. At any desired time in the dynamics various observables are measured, such as the $x$ magnetization, correlation functions, or the projection onto specific states, enabling a detailed study of the DQPT.

To account for the ground-state degeneracy of $H_{0}$, while still allowing us to connect to other relevant observables as we show later on, the Loschmidt amplitude $\mathcal{G}(t)$ is replaced by the probability to return to the ground-state manifold after a time $t, P(t)=P_{\Rightarrow}(t)+P_{\models}(t)[22,31]$. As for $\mathcal{G}(t)$, we introduce the rate function

$$
\Lambda(t)=-N^{-1} \log [P(t)] .
$$

At the critical time $t_{c}$ of a DQPT, $\Lambda(t)$ becomes nonanalytic.

We can understand the origin of this nonanalyticity by noticing that, for $N \rightarrow \infty, \Lambda(t)$ is completely dominated by either $P_{\Rightarrow}(t)$ or $P_{\models}(t)$, as illustrated in the inset of Fig. 2(a) for $N \leq 10$ [see also Fig. S1(a) [24]]. At the critical time $t=t_{c}$, the dominant probability switches from $P_{\Rightarrow}(t)$ to $P_{\models}(t)$, implying a discontinuous behavior for large $N$. A useful tool to extract this nonanalytic behavior already for small systems is given by $[22,31]$

$$
\lambda(t) \equiv \min _{\eta \in\{\Rightarrow, \Leftarrow\}}\left(-N^{-1} \log \left[P_{\eta}(t)\right]\right),
$$



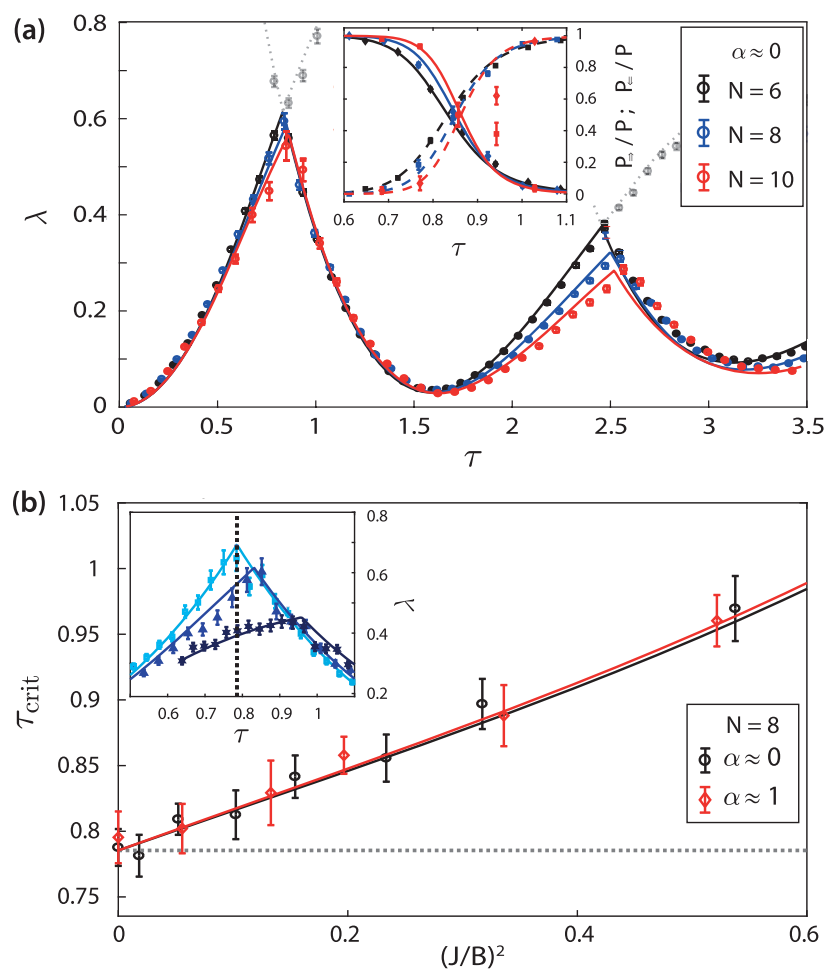

FIG. 2. Observation of dynamical quantum phase transition. (a) Measured rate function $\lambda(\tau)$ for three different system sizes at $J / B \approx 0.42$, showing a nonanalytical behavior (with $\tau=t B$ being the dimensionless time). Dots are experimental data with error bars estimated from quantum projection noise; lines are numerical simulations with experimental parameters. In a lighter black color we have included data for the subdominant contributions at $N=6$. Inset: The transition between the normalized ground-state probabilities $P_{\Rightarrow} / P$ (solid lines) and $P_{\models} / P$ (dashed lines) becomes sharper for larger $N$. (b) The first-order correction to the critical time $\tau_{\text {crit }}$, i.e., the occurrence of the first DQPT, is linear as a function of $(J / B)^{2}$ for small $J / B$, and approximately independent of interaction range. Error bars are $1 \sigma$ confidence intervals of the fits on $\log [P \Rightarrow, \Leftarrow(\tau)]$ from which we extract $\tau_{\text {crit }}$ (see Supplemental Material [24]). Inset: DQPT for $(J / B)=0$, 0.392 , and 0.734 (light blue, dark blue, and black dots). The grey dashed lines indicate $\tau_{\text {crit }}$ for $(J / B)=0$.

which coincides with $\Lambda$ for large $N$, and which we use in our further discussions of the DQPTs.

In Fig. 2(a), we report our first main result, the direct observation of a DQPT through nonanalyticities in the rate function $\lambda$. Let us emphasize that the observed DQPTs in $\lambda$ are neither artificially caused by our definition of $P(t)$ nor by the resulting minimum construction. The definition $P(t)=P_{\Rightarrow}(t)+P_{\models}(t)$ is physically motivated [22,31] by allowing us to connect the DQPTs to other physical quantities, as we demonstrate also below. The rate function $\lambda$, on the other hand, provides a tool to quantitatively extract the DQPT already for small systems, resulting in very weak residual finite-size corrections [24], such that we can focus in the following on a single system size. Without the minimum construction the DQPT has to be determined via the sharpening of the finite-size crossover for increasing system size, which is much more intricate [24]. While in the following we concentrate our discussion mainly on the first DQPT, let us emphasize that also the subsequent DQPTs are of the same nature, possibly with the role of $P \Rightarrow(t)$ and $P_{\models}(t)$ exchanged.

To study the robustness of DQPTs against deformations of the Hamiltonian, we extract the first critical time $t_{c}$ from $\lambda(t)$ as a function of the coupling strength $J=(N-1)^{-1}$ $\sum_{i>j} J_{i j}$; see Fig. 2(b). We find that the temporal nonanalytic behavior is stable over a broad range of $J / B$ and for different $\alpha$. For $J / B \ll 1$, the critical time $\tau_{c}-\pi / 4 \propto$ $(J / B)^{2}$ exhibits a quadratic dependence on $J / B$ yielding $\tau_{c}=\pi / 4$ for $J=0$ where the dynamics becomes equivalent to Larmor precession of $N$ independent spins. While DQPTs also appear in this apparently simple case, it is important to emphasize that $J=0$ represents a singular point in the dynamics due to the absence of nonlocal quantum fluctuations as becomes apparent from the entanglement dynamics we discuss later on.

We now present measurements that connect DQPTs to other observables, further corroborating the theory of DQPT as a key framework for understanding quantum many-body dynamics. In Figs. 3(a) and 3(b), we compare $\lambda(t)$ and the evolution of the magnetization, $M_{x}(t)=$ $\left\langle\mathcal{M}_{x}(t)\right\rangle$ with $\mathcal{M}_{x}=N^{-1} \sum_{i} \sigma_{i}^{x}$. The initial state breaks the global $\mathbb{Z}_{2}$ symmetry $\sigma_{i}^{x} \rightarrow-\sigma_{i}^{x} \forall i$ of the Hamiltonian $H$. The system responds to this symmetry breaking by a repeated crossover between the $M_{x}>0$ and $M_{x}<0$ sectors, reaching the symmetry-restoring value $M_{x}=0$ at specific times. Comparing with $\lambda(t)$, these are tied to the critical times of the DQPT, whose essence is the symmetry restoration in the ground-state manifold.

This connection is tightened by resolving the magnetization $M_{x}(\varepsilon, t)$ as a function of energy density $\varepsilon$ (see Supplemental Material [24], and Ref. [31]), where $\varepsilon=$ $E / N$ and $E$ is the energy measured with the initial Hamiltonian $H_{0}$. The measured data are displayed in Fig. 3(c). The dynamics along $\varepsilon=0$ (ground-state manifold) is directly understood from the previous discussion. In large systems, as long as $t<t_{c}$ one has $P(t) \approx P_{\Rightarrow}(t)$, yielding $M_{x}\left(\varepsilon=0, t<t_{c}\right) \approx 1$. For $t>t_{c}, P_{\models}(t)$ takes over, and $M_{x}(\varepsilon=0, t)$ jumps to -1 . With increasing energy densities this sudden change smears out. Its influence, however, persists up to the system's mean energy density $\bar{\varepsilon}(t)$ [solid line in Fig. 3(c)], where observables such as $M_{x}(t)$ acquire their dominant contribution [31]. In this way, as sketched in Fig. 1, an extended region of the dynamics is controlled by the DQPT, reminiscent of a quantum critical region at an equilibrium QPT.

As the final result of our work, we now show that DQPTs in the simulated Ising models also control entanglement production. In this way, we connect entanglement as an important concept for the characterization of equilibrium phases and criticality [32] to DQPTs. In Fig. 4(a), we show 

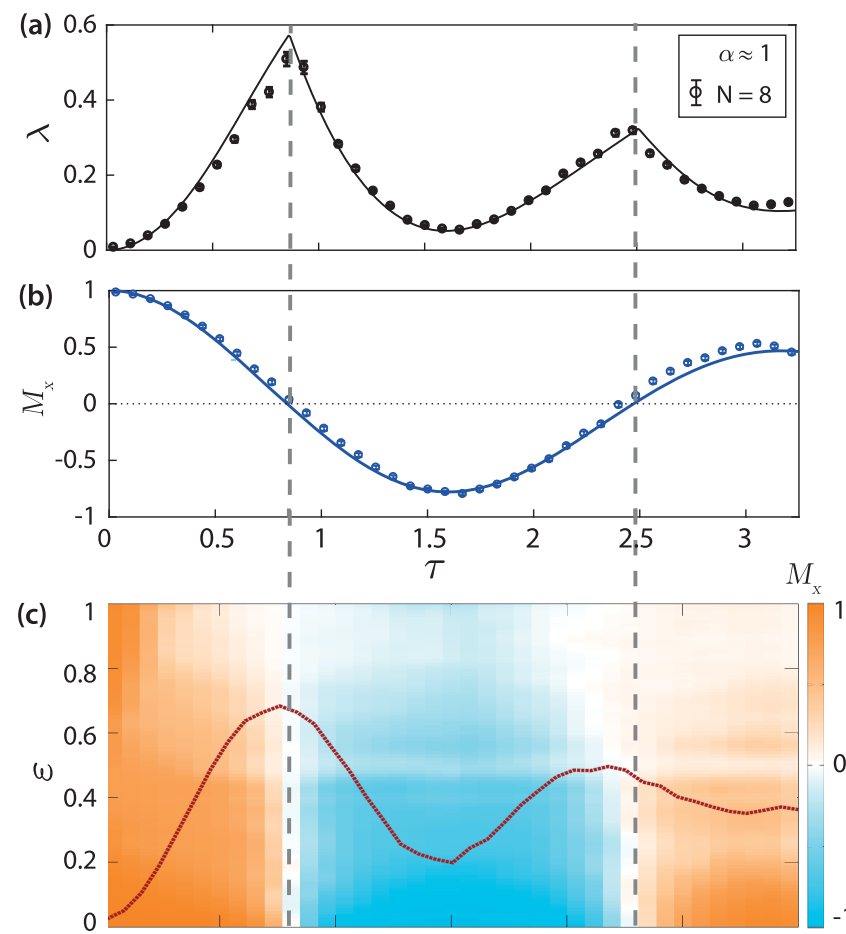

FIG. 3. Control of the magnetization dynamics by a DQPT. DQPTs, indicated by kinks in $\lambda(\tau)$ (a), control the average magnetization in the $x$ direction, $M_{x}$ (b). (c) This connection becomes apparent when resolving the magnetization against energy density $\epsilon$, with the nonanalyticity at $\epsilon=0$ radiating out to $\epsilon>0$. For details on the measurement of the energy-resolved magnetization, see Supplemental Material [24]. In (a) and (b), dots indicate experimental data with errors derived from quantum projection noise; solid lines denote numerical simulations $(J / B=0.5)$.

the half-chain entropy $\mathcal{S}(t)$ measured by quantum tomography (see Supplemental Material [24]). $\mathcal{S}(t)$ exhibits its strongest growth in the vicinity of a DQPT. While these data are suggestive of entanglement production, $\mathcal{S}(t)$ is an entanglement measure only for pure states, which does not account for the experimentally inevitable mixing caused by decoherence. Therefore, we additionally measure a mixedstate entanglement witness, the Kitagawa-Ueda spinsqueezing parameter $\xi_{s}$ [33] (see Supplemental Material [24]) signaling entanglement whenever $\xi_{s}<1$. As Fig. 4(b) shows, $\xi_{s}$ presents a behavior qualitatively very similar to $\mathcal{S}(t)$. Related to common spin-squeezing scenarios [34], the spin squeezing is most effective when the mean spin vector on the Bloch sphere is perpendicular to the direction of the spin-spin interaction. Importantly, this occurs when $M_{x}=0$, which we found above to be inherently tied to DQPTs. The presence of the DQPT, moreover, offers a more general interpretation: At exactly $t_{c}$, the ground-state manifold enters the equal superposition $(|\Rightarrow\rangle+|\Leftarrow\rangle) / \sqrt{2}$, a highly entangled Greenberger-Horne-Zeilinger (GHZ) state. Just as for the case of $M_{x}$, our data suggest that the influence of this state stretches to elevated energy densities, and thus DQPTs (a)
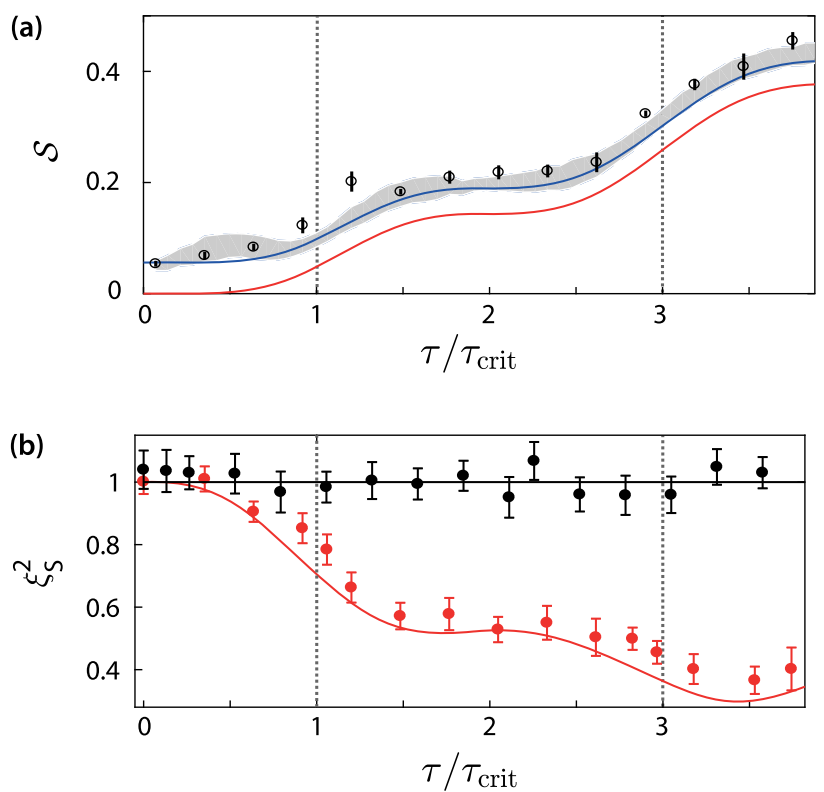

FIG. 4. Entanglement production. Dynamics of (a) the halfchain entropy $\mathcal{S}$ and (b) spin squeezing $\xi_{S}^{2}$ for $N=6$ spins at $\alpha \approx 0$. For nonzero interactions, both entanglement quantifiers show a marked increase in the vicinity of the DQPTs, indicated by dashed lines $[J / B=0.223$ in (a) and 0.25 in (b) $]$. (a) Comparison of the measured half-chain entropy obtained from quantum tomography (circles) with the entropies resulting from solving the Schrödinger equation using our experimental parameters, with the ideal input state $|\Rightarrow\rangle$ (red line) and a slightly depolarized input state (blue line). Entropies obtained from simulating the tomographic reconstruction including projection noise are slightly higher, as indicated for the mixed initial state by the shaded area ( $1 \sigma$ confidence region). (b) The change in $\xi_{s}^{2}(t)$ signals qualitatively similar entanglement production (red symbols). For $J / B=0$, no entanglement is created (black symbols).

control also entanglement production. Numerical simulations show qualitatively no difference for $\alpha>0$, suggesting that these features are independent of the interaction range.

We have presented the first direct observation of dynamical quantum phase transitions by revealing temporal nonanalyticities in physical quantities, measured in a system of trapped ions. We have demonstrated how the nonanalytic behavior provides a unifying principle of quantum many-body dynamics, governing the real-time evolution of other observables such as the magnetization and entanglement production, similar to the way that nonanalyticities in the free energy determine the behavior of other observables in equilibrium phase transitions. While we have studied a specific model system, our methodology can be applied in a much more general context, and is potentially applicable also to other nonequilibrium phenomena such as many-body localization $[3,4]$ or quantum time crystals $[35,36]$.

This work was supported by the Austrian Science Fund (FWF) under Grants No. P25354-N20 and No. F4016-N23 
(SFB FoQuS), the European Research Council (ERC) Synergy Grant No. UQUAM, the Deutsche Akademie der Naturforscher Leopoldina under Grant No. LPDR 2015-01, the Deutsche Forschungsgemeinschaft via the Gottfried Wilhelm Leibniz Prize program, and the Institut für Quanteninformation $\mathrm{GmbH}$.

*Present address: ARC Centre of Excellence for Engineered Quantum Systems, School of Physics, University of Sydney, New South Wales 2006, Australia.

[1] J. Eisert, M. Friesdorf, and C. Gogolin, Nat. Phys. 11, 124 (2015).

[2] T. Langen, R. Geiger, and J. Schmiedmayer, Annu. Rev. Condens. Matter Phys. 6, 201 (2015).

[3] M. Schreiber, S. S. Hodgman, P. Bordia, H. P. Lüschen, M. H. Fischer, R. Vosk, E. Altman, U. Schneider, and I. Bloch, Science 349, 842 (2015).

[4] J. Smith, A. Lee, P. Richerme, B. Neyenhuis, P. W. Hess, P. Hauke, M. Heyl, D. A. Huse, and C. Monroe, Nat. Phys. 12, 907 (2016).

[5] M. Gring, M. Kuhnert, T. Langen, T. Kitagawa, B. Rauer, M. Schreitl, I. Mazets, D. A. Smith, E. Demler, and J. Schmiedmayer, Science 337, 1318 (2012).

[6] B. Neyenhuis, J. Smith, A. C. Lee, J. Zhang, P. Richerme, P. W. Hess, Z. X. Gong, A. V. Gorshkov, and C. Monroe, arXiv:1608.00681.

[7] E. A. Martinez, C. A. Muschik, P. Schindler, D. Nigg, A. Erhard, M. Heyl, P. Hauke, M. Dalmonte, T. Monz, P. Zoller et al., Nature (London) 534, 516 (2016).

[8] D. Fausti, R. I. Tobey, N. Dean, S. Kaiser, A. Dienst, M. C. Hoffmann, S. Pyon, T. Takayama, H. Takagi, and A. Cavalleri, Science 331, 189 (2011).

[9] M. Heyl, A. Polkovnikov, and S. Kehrein, Phys. Rev. Lett. 110, 135704 (2013).

[10] S. Sachdev, Quantum Phase Transitions (Cambridge University Press, Cambridge, 2011).

[11] A. LeClair, G. Mussardo, H. Saleur, and S. Skorik, Nucl. Phys. B453, 581 (1995).

[12] M. Heyl, Phys. Rev. Lett. 115, 140602 (2015).

[13] C. Karrasch and D. Schuricht, Phys. Rev. B 87, 195104 (2013).

[14] J. N. Kriel, C. Karrasch, and S. Kehrein, Phys. Rev. B 90, 125106 (2014).
[15] E. Canovi, P. Werner, and M. Eckstein, Phys. Rev. Lett. 113, 265702 (2014).

[16] J. C. Budich and M. Heyl, Phys. Rev. B 93, 085416 (2016).

[17] S. Sharma, U. Divakaran, A. Polkovnikov, and A. Dutta, Phys. Rev. B 93, 144306 (2016).

[18] N. Fläschner, D. Vogel, M. Tarnowski, B. S. Rem, D.-S. Lühmann, M. Heyl, J. C. Budich, L. Mathey, K. Sengstock, and C. Weitenberg, arXiv:1608.05616.

[19] D. Porras and J. I. Cirac, Phys. Rev. Lett. 92, 207901 (2004).

[20] K. Kim, M. S. Chang, R. Islam, S. Korenblit, L. M. Duan, and C. Monroe, Phys. Rev. Lett. 103, 120502 (2009).

[21] P. Jurcevic, B. P. Lanyon, P. Hauke, C. Hempel, P. Zoller, R. Blatt, and C. F. Roos, Nature (London) 511, 202 (2014).

[22] B. Zunkovic, M. Heyl, M. Knap, and A. Silva, arXiv: 1609.08482 .

[23] J. C. Halimeh and V. Zauner-Stauber, arXiv:1610.02019.

[24] See Supplemental Material http://link.aps.org/supplemental/ 10.1103/PhysRevLett.119.080501 for experimental and theoretical details, which includes Refs. [25-30].

[25] P. Schindler, D. Nigg, T. Monz, J. T. Barreiro, E. Martinez, S. X. Wang, S. Quint, M. F. Brandl, V. Nebendahl, C. F. Roos et al., New J. Phys. 15, 123012 (2013).

[26] A. Sørensen and K. Mølmer, Phys. Rev. Lett. 82, 1971 (1999).

[27] J. W. Britton, B. C. Sawyer, A. C. Keith, C. C. Wang, J. K. Freericks, H. Uys, M. J. Biercuk, and John J. Bollinger, Nature (London) 484, 489 (2012).

[28] R. Islam, C. Senko, C. Campbell, S. Korenblit, J. Smith, A. Lee, E. E. Edwards, C.-C. J. Wang, J. K. Freericks, and C. Monroe, Science 340, 583 (2013).

[29] P. Nevado and D. Porras, Phys. Rev. A 93, 013625 (2016).

[30] M. Kac, G. E. Uhlenbeck, and P. C. Hemmer, J. Math. Phys. (N.Y.) 4, 216 (1963).

[31] M. Heyl, Phys. Rev. Lett. 113, 205701 (2014).

[32] N. Laflorencie, Phys. Rep. 646, 1 (2016).

[33] M. Kitagawa and M. Ueda, Phys. Rev. A 47, 5138 (1993).

[34] L. Pezzè, A. Smerzi, M. K. Oberthaler, R. Schmied, and P. Treutlein, arXiv:1609.01609.

[35] J. Zhang, P. W. Hess, A. Kyprianidis, P. Becker, A. Lee, J. Smith, G. Pagano, I. D. Potirniche, A. C. Potter, A. Vishwanath et al., Nature (London) 543, 217 (2017).

[36] S. Choi, J. Choi, R. Landig, G. Kucsko, H. Zhou, J. Isoya, F. Jelezko, S. Onoda, H. Sumiya, V. Khemani et al., Nature (London) 543, 221 (2017). 